\title{
Detection and Analysis of Change in Remotely Sensed Imagery with Application to Wide Area Surveillance
}

\author{
Mark J. Carlotto, Senior Member, IEEE
}

\begin{abstract}
A new approach to wide area surveillance is described that is based on the detection and analysis of changes across two or more images over time. Methods for modeling and detecting general patterns of change associated with construction and other kinds of activities that can be observed in remotely sensed imagery are presented. They include a new nonlinear prediction technique for measuring changes between images and temporal segmentation and filtering techniques for analyzing patterns of change over time. These methods are applied to the problem of detecting facility construction using Landsat Thematic Mapper imagery. Full scene results show the methods to be capable of detecting specific patterns of change with very few false alarms. Under all conditions explored, as the number of images used increases, the number of false alarms decreases dramatically without affecting the detection performance. It is argued that the processing gain that results in using more than two images justifies the increased computational complexity and storage requirements of our approach over single image object detection and conventional change detection techniques.
\end{abstract}

\section{INTRODUCTION}

A $\mathrm{N}$ IMPORTANT area in wide-area surveillance (WAS) is the detection of new activities and events (e.g., facility construction/demolition, deforestation, flooding, etc.) over very large geographic areas. Since they are new (i.e., not previously captured in a database), site models do not yet exist, and model-supported approaches [1] cannot be used. Automatic target recognition (ATR), which is focused primarily on vehicle detection, is not well-suited to the problem of detecting new activities and events in imagery because it is usually not possible to specify a specific geometrical model of the activity in advance. Historically, alternative approaches (e.g., single image manmade object detection using fractals [2]-[3], simple change detection techniques [4]-[9], and even contextual approaches that use terrain data to optimize detection algorithms for different parts of the scene and to eliminate false alarms in unlikely areas) have been unable to achieve the required detection rates while maintaining an acceptable level of false alarms.

In this paper, we describe a new method for detecting patterns of change associated with construction and other kinds of activities that can be observed in remotely sensed imagery. Our approach is based on modeling the changes that are expected to be observed in imagery corresponding to the

Manuscript received November 1, 1995; revised July 30, 1996.

The author is with Pacific-Sierra Research Corporation, Arlington, VA 22209 USA (e-mail: markc@sed.psrw.com).

Publisher Item Identifier S 1057-7149(97)00602-7. different phases of the activity over time. For example, during different phases of facility construction, the reflectance of the surface undergoes distinct changes. In visible wavelengths, as vegetation is cleared, the surface reflectance increases significantly. Then, as the surface cover is scraped away and excavation begins, the reflectance changes again as bare soil and rock are exposed. The spectral reflectance further increases as concrete footings are poured and the structure begins to take shape. Unlike vegetation, which follows relatively welldefined spectral trajectories over time [10]-[11], man-made changes are more difficult to model in precise terms. In developing a model for facility construction, it must allow for a certain amount of uncertainty in time (since construction schedules may vary) and in space (actual size and shape of facility are generally unknown ahead of time). In our approach, patterns of change are expressed in terms of the relative values of image properties (e.g., brightness, temperature, biomass estimates, etc.) over time. By using relative values, we can describe changes in terms of general trends (e.g., the increase in surface reflectance associated with a new construction activity).

Section II reviews related work in change detection and multitemporal processing techniques. Section III describes our approach. Experimental results are presented in Section IV. Conclusions and areas for future work are discussed in Section $\mathrm{V}$.

\section{RELATED WORK}

Our approach is related to previous work in change detection and multitemporal image analysis. An early discussion of change detection can be found in Rosenfeld [4]. Early change detection techniques were based on statistical measures of similarity between images such as cross correlation and entropy [5]. These techniques required the images to be coregistered. This then motivated the development of symbolic techniques that detected changes by segmenting and matching regions in terms of their size, shape, spectral properties, and spatial relations [6]. Other work that led to our approach include linear prediction [7], adaptive subtraction [8], and the perpendicular change index [9]. Like the early change-detection techniques, the techniques described in this paper also require the input images to be registered to each other.

Multitemporal techniques involve the analysis of two or more images. Some methods simply treat multitemporal data 

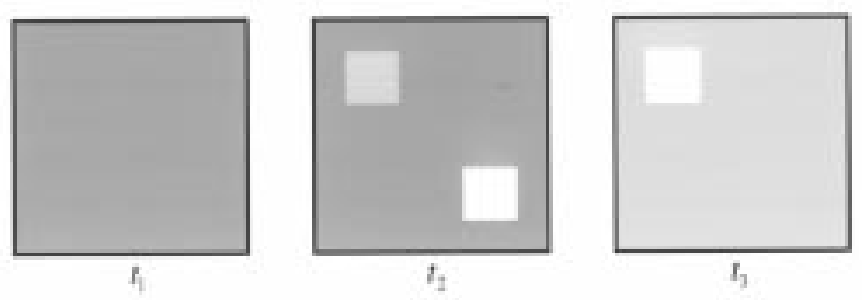

(a)

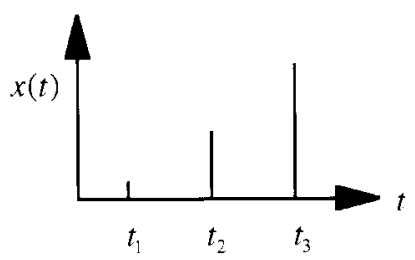

(b)

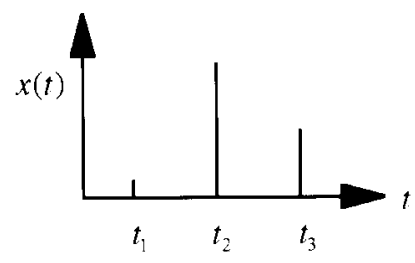

(c)
Fig. 1. Events captured in imagery over time exhibit different patterns of change. (a) Two events captured in three images. (b) Pattern of change (upper left). (c) Pattern of change (lower right).

as features for classification. For example, Byrne et al. [12] perform a principal components analysis of Landsat data and show that gross differences in overall radiation and atmospheric changes appear in the major component images and that changes in land cover appear in the minor component images. Others explicitly model changes in physically significant features such as the tasseled cap greenness [13] over time. The Delta classifier [10] was an early method developed for classifying vegetation based on general growth state models. More recent methods based on parameterized temporal profiles [11] have been successfully applied over large regions for crop classification and for estimating crop status and key phenological events related to crop yield.

\section{Methodology}

\section{A. Overview}

Change detection techniques determine areas in a scene that have changed in some way but cannot in general differentiate between different kinds of changes. Consider an example (Fig. 1) where three images acquired at times $t_{1}, t_{2}$, and $t_{3}$ have captured two events (Fig. 1(a)). The event in the upper left corner appears in the second image and increases in brightness from the first to the third image (Fig. 1(b)). The event in the lower right corner is a transient change. It also appears in the second image as an increase in brightness but disappears in the third image (Fig. 1(c)). In addition, notice that the background in the third image is somewhat brighter than the other two images. The two events are the same size but have distinctly different patterns of change over time. A pattern of change can be thought of as the variation in the value of an observable such as image brightness as a function of time, e.g., $x\left(t_{1}\right)<x\left(t_{2}\right)<x\left(t_{3}\right)$. Our approach to WAS is based on detecting or enhancing patterns of change in imagery that are observed when specific kinds of events or activities occur. Where change detection detects any significant

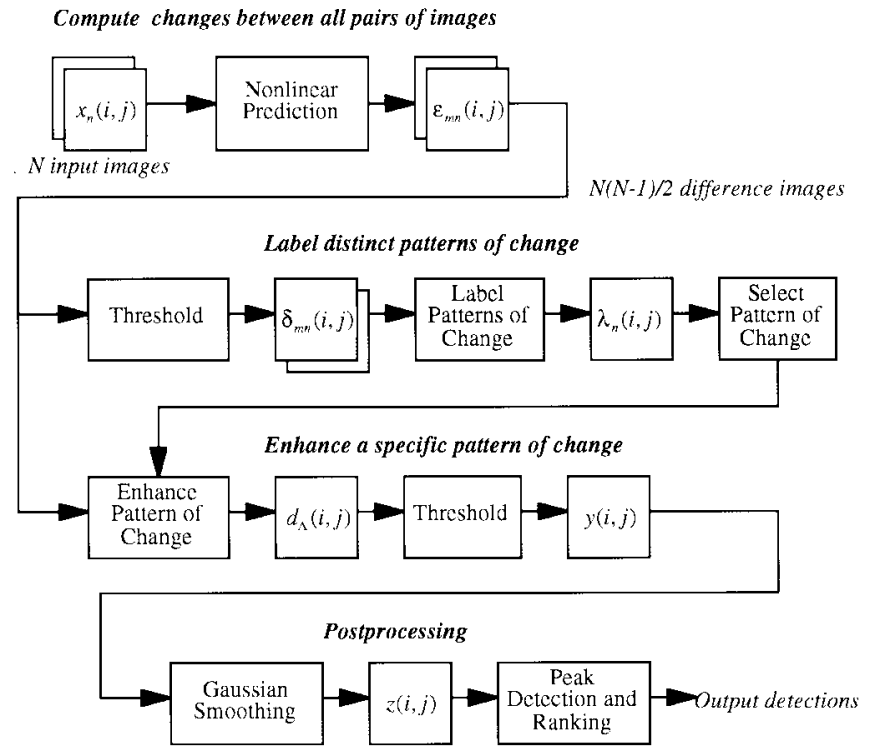

Fig. 2. Overview of change detection and analysis methodology.

change between pairs of images, our method extracts only those changes that match a given pattern over time.

Fig. 2 gives an overview of our methodology. Due to changes in solar angle, sensor gain, atmospheric scattering, path radiance, environmental conditions, and other factors, patterns of change are not derived directly from the sensed brightness values. Instead, we compute a measure of change between images that is less sensitive to the above factors, and from these changes, we infer the pattern of change indirectly. Section III-B formulates the measurement of change as a prediction problem. Both linear and nonlinear prediction techniques are considered. The set of change images computed between all pairs of input images is then processed to identify or enhance particular patterns of change over time. Two temporal analysis techniques are discussed. In the first technique (Section III-C), each of the difference images is thresholded to produce a set of binary images. From the set of binary images, a label image is produced. Each label corresponds to a unique pattern of change. We show that the performance of this technique depends critically on the threshold values used. As a result, it is more useful as a data analysis technique - to provide insight into the kinds of change that have occurred-rather than as a detection technique. In the second technique, instead of thresholding the difference images, they are combined by a filter that emphasizes a particular pattern of change. This pattern may have been identified using the first technique or have been specified by an image analyst. The output of the filter is thresholded to produce a binary image. The resultant binary image is smoothed by a Gaussian that is about the size of the expected change. Peaks locations are extracted, rank-ordered in terms of their magnitude, and used to cue an image analyst to areas in the imagery likely to contain changes of interest.

\section{B. Techniques for Measuring Change}

In simple image subtraction, the measure of change is based only on the difference between corresponding pixel values. 


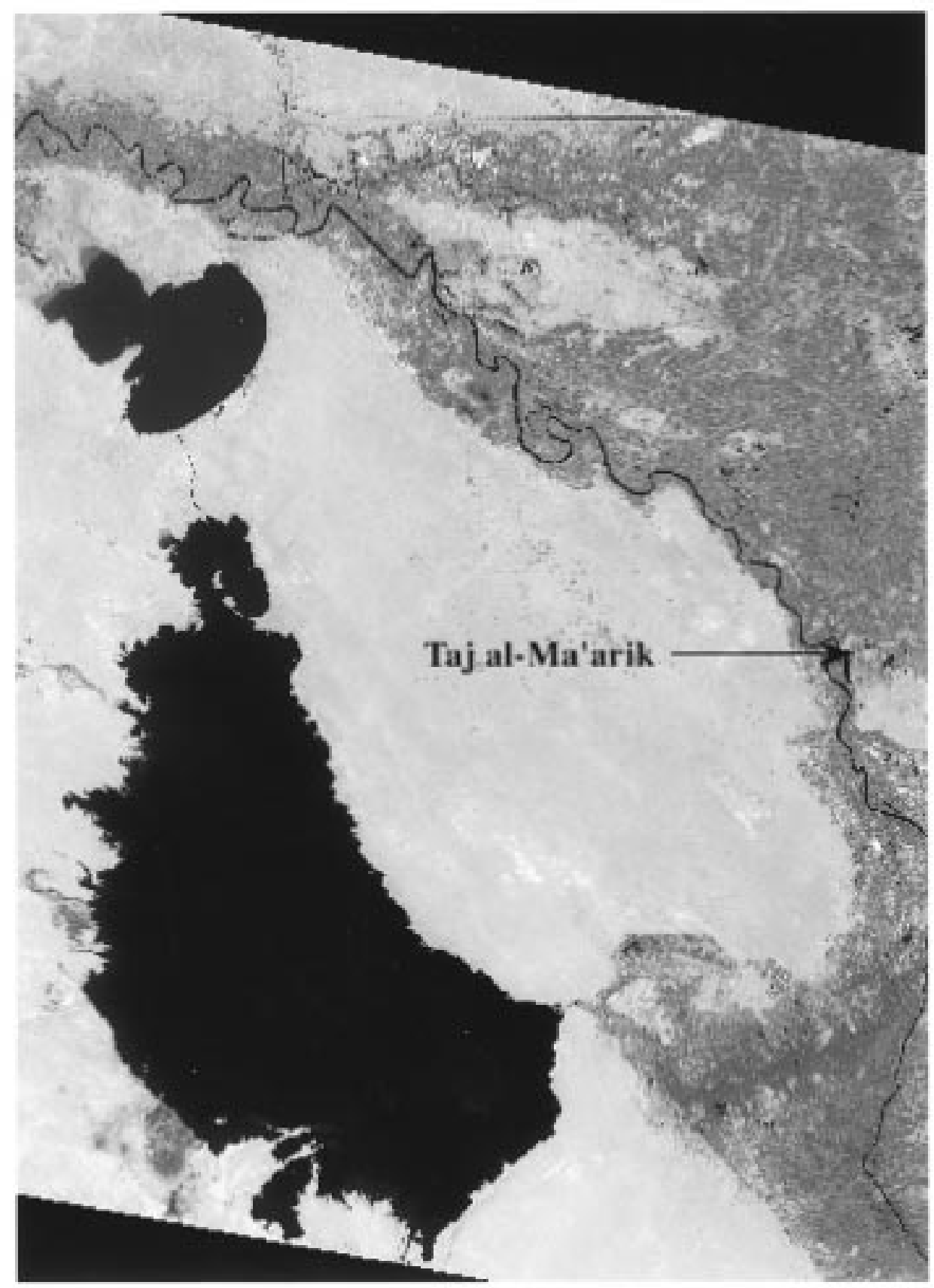

Fig. 3. Landsat image over region of interest (7/10/90).

Adaptive techniques use information over larger areas (e.g., within a sliding window) to model and predict two or more images from one another. The difference between the actual and predicted image is used as a measure of change. For two images (random variables) $x_{1}(i, j)$ and $x_{2}(i, j)$ acquired at times $t_{1}$ and $t_{2}$, where $t_{2}>t_{1}$, we seek an estimate of $x_{2}$ based on $x_{1}$, i.e., $\hat{x}_{2}=f_{21}\left(x_{1}\right)$, that minimizes the mean-square error (MSE)

$$
\begin{aligned}
\varepsilon_{F} & =E\left[\left(x_{2}-\hat{x}_{2}\right)^{2}\right]=E\left[\left(x_{2}-f_{21}\left(x_{1}\right)\right)^{2}\right] \\
& =\int_{-\infty}^{\infty} \int_{-\infty}^{\infty}\left(x_{2}-f_{21}\left(x_{1}\right)\right)^{2} p\left(x_{1}, x_{2}\right) d x_{1} d x_{2}
\end{aligned}
$$

where $\varepsilon_{F}$ is the forward prediction error. Similarly, in the other direction, we seek an estimate of $x_{1}$ based on $x_{2}$, i.e., $\hat{x}_{1}=f_{12}\left(x_{2}\right)$, that minimizes the backward prediction error

$$
\begin{aligned}
\varepsilon_{B} & =E\left[\left(x_{1}-\hat{x}_{1}\right)^{2}\right]=E\left[\left(x_{1}-f_{12}\left(x_{2}\right)\right)^{2}\right] \\
& =\int_{-\infty}^{\infty} \int_{-\infty}^{\infty}\left(x_{1}-f_{12}\left(x_{2}\right)\right)^{2} p\left(x_{1}, x_{2}\right) d x_{1} d x_{2} .
\end{aligned}
$$

When the functions $f_{12}$ and $f_{21}$ are linear, the parameters of the forward and backward predictors

$$
\begin{aligned}
& f_{21}\left(x_{1}\right)=a x_{1}+b \\
& f_{12}\left(x_{2}\right)=c x_{2}+d
\end{aligned}
$$

are obtained using linear regression. In the linear prediction model, the gains and offsets adjust for global differences between the two images (e.g., due to solar angle, sensor 


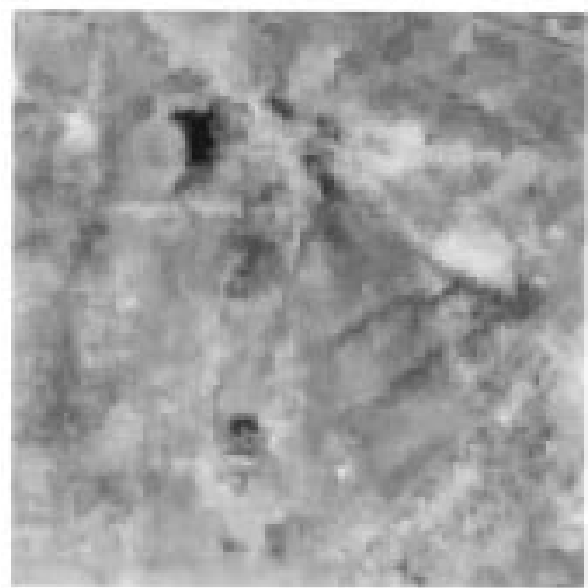

(a)

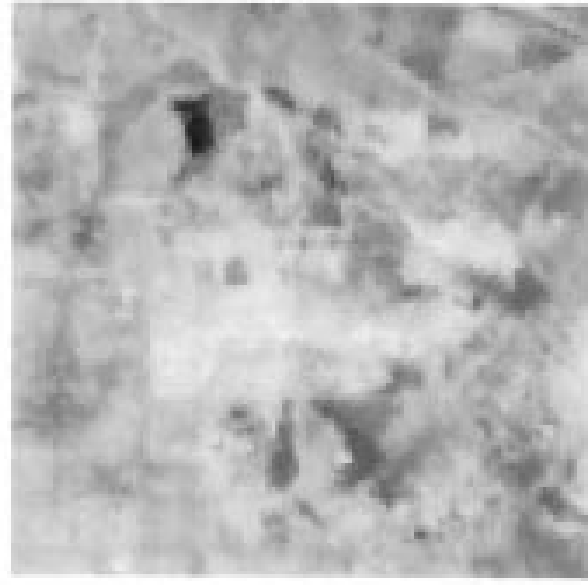

(c)

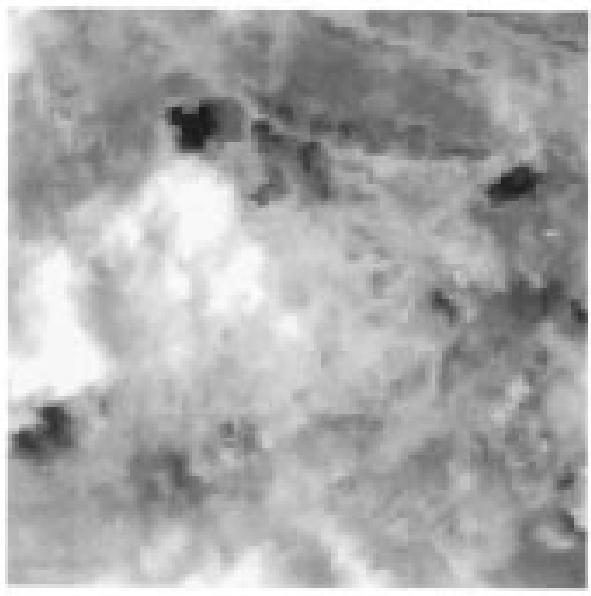

(e)

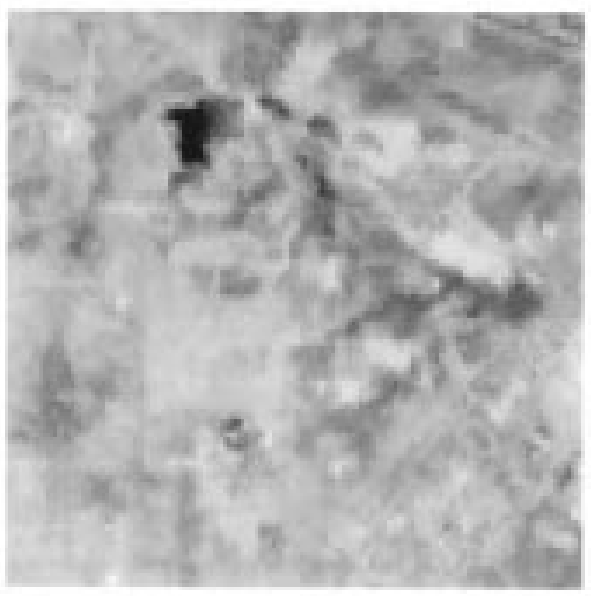

(b)

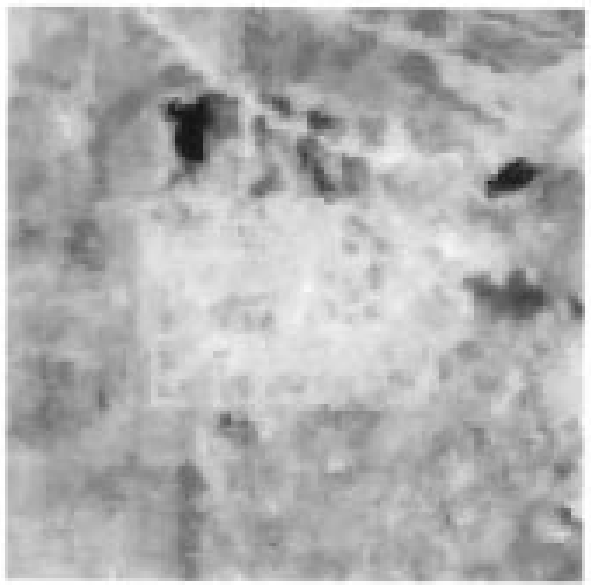

(d)

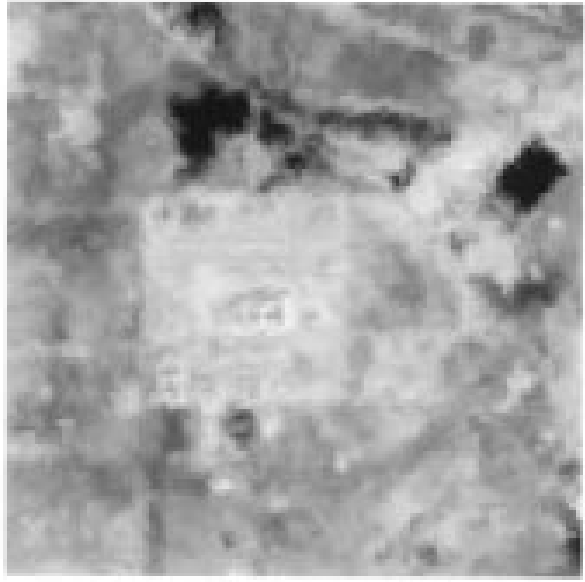

(f)

Fig. 4. Phases of construction at Taj al-Ma'arik. (a) Pre-construction (1/15/87). (b) Early-construction (4/21/87). (c) Early-construction (6/24/87). (d) Mid-construction (3/30/88). (e) Cloud cover (5/1/88). (f) Construction complete (3/4/90).

gain, atmospheric transmission, scattering, and changes in the background). As demonstrated in Section IV, the linear model works well in detecting changes when the amount of change is small. However, in situations where there are large changes, e.g., due to clouds or significant seasonal variations, the linear model, in attempting to adapt to these changes as if they were part of the background, tends to introduce spurious changes that ultimately lead to false alarms. 
This motivates the development of a more general model that can handle cases where there may be large changes between images. In particular, we allow $f_{12}$ and $f_{21}$ to be nonlinear functions of image brightness. In the general case, it can be shown that the MSE is minimized when these functions are the conditional expected values [14]

$$
\begin{aligned}
f_{21}\left(x_{1}\right) & =\int_{-\infty}^{\infty} x_{2} p\left(x_{2} \mid x_{1}\right) d x_{2} \\
f_{12}\left(x_{2}\right) & =\int_{-\infty}^{\infty} x_{1} p\left(x_{1} \mid x_{2}\right) d x_{1}
\end{aligned}
$$

where $p\left(x_{1} \mid x_{2}\right) p\left(x_{2}\right)=p\left(x_{2} \mid x_{1}\right) p\left(x_{1}\right)=p\left(x_{1}, x_{2}\right)$ is the joint density, and $p\left(x_{1}\right)$ and $p\left(x_{2}\right)$ are the marginals.

The forward and backward difference images are

$$
\begin{aligned}
& \varepsilon_{F}(i, j)=x_{2}(i, j)-f_{21}\left(x_{1}(i, j)\right), \\
& \varepsilon_{B}(i, j)=x_{1}(i, j)-f_{12}\left(x_{2}(i, j)\right) .
\end{aligned}
$$

The forward difference image is greater than (less than) zero where there is an increase (decrease) in brightness in the later image that cannot be modeled in terms of the earlier image. The backward difference image is greater than (less than) zero where there is an decrease (increase) in brightness in the earlier image that cannot be modeled in terms of the later image. The total difference image $\varepsilon=\varepsilon_{F}(i, j)-\varepsilon_{B}(i, j)$ will be greater than zero either when a dark object disappears or a bright object appears and will be less than zero when a dark object appears or a bright object disappears.

Our approach to WAS involves the analysis of changes between all pairs of input images. For $N$ input images $\left\{x_{n}(i, j), 0 \leq n<N\right\}$, there are $N(N-1) / 2$ total difference images $\left\{\varepsilon_{m n}(i, j), 0 \leq m<n, 0<n<N\right\}$, where the total difference between the $m$ th and $n$th input images $\left(t_{m}<t_{n}\right)$ is

$$
\begin{aligned}
\varepsilon_{m n}(i, j)= & {\left[x_{n}(i, j)-f_{n m}\left(x_{m}(i, j)\right)\right] } \\
& -\left[x_{m}(i, j)-f_{m}\left(x_{n}(i, j)\right)\right] .
\end{aligned}
$$

It can be shown that $\varepsilon_{m n}(i, j)=\varepsilon_{m n^{\prime}}(i, j)+\varepsilon_{n^{\prime} n}(i, j)$ only when there is a one-to-one mapping between the $x_{m}, x_{n}$, and $x_{n^{\prime}}$ brightness values. In this case, one of the three difference images is redundant.

Finally, it is noted that the nonlinear prediction technique can also be applied to multiband images [15], i.e., to predict two vectors of random variables (spectral bands) $\boldsymbol{x}_{m}=$ $\left[x_{m, 1} \cdots x_{m, B}\right]$ and $\boldsymbol{x}_{n}=\left[x_{n, 1} \cdots x_{n, B}\right]$ from each other:

$$
\begin{aligned}
\hat{x}_{m, b} & =f_{m n, b}\left[x_{n, 1} \cdots x_{n, B}\right] \\
\hat{x}_{n, b} & =f_{n m, b}\left[x_{m, 1} \cdots x_{m, B}\right]
\end{aligned}
$$

where $B$ is the number of bands.

\section{Temporal Segmentation}

Temporal segmentation extracts unique patterns of change across images over time. The total difference images (6) are thresholded to produce binary change images

$$
\delta_{m n}(i, j)= \begin{cases}1, & \left|\varepsilon_{m n}(i, j)\right|>\tau_{m n} \\ 0, & \text { otherwise }\end{cases}
$$

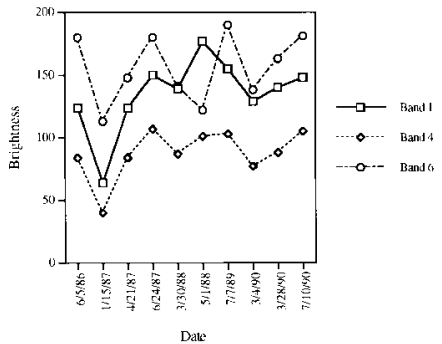

Fig. 5. Plots of bands 1, 4, and 6 brightness over Taj al-Ma'arik site versus time.

The $\tau_{m n}$ thresholds are computed from the histograms of the total difference images. Each is chosen to satisfy

$$
p_{0}=\sum_{|\varepsilon|>\tau_{m n}} p_{m n}(\varepsilon)
$$

where $p_{m n}(\varepsilon)$ is the histogram of the total difference image $\varepsilon_{m n}(i, j)$, and $p_{0}$ is the fraction of the image that is assumed to have changed.

Patterns of change are represented by an image of label vectors $\Lambda(i, j)=\left[\lambda_{n}(i, j)\right]$. At a particular pixel location, two elements of the label vector are equal $\lambda_{m}(i, j)=\lambda_{n}(i, j)$ if there is no change between the corresponding times at that pixel $\delta_{m n}(i, j)=0$; otherwise, the two elements are different $\lambda_{m}(i, j) \neq \lambda_{n}(i, j)$. The following algorithm is used to compute the image of label vectors from the set of binary change images:

$$
\begin{aligned}
& \text { For each }(i, j) \text { : } \\
& \text { Let } \lambda_{n}(i, j)=n, \text { for } n=1,2, \cdots, N \\
& \text { For } n=1 \text { to } N-1 \text { : } \\
& \quad \text { For } m=0 \text { to } n-1 \text { : } \\
& \quad \text { If } \delta_{m n}(i, j)=0, \text { Then } \lambda_{m}(i, j), \lambda_{n}(i, j) \\
& \quad=\min \left\{\lambda_{m}(i, j), \lambda_{n}(i, j)\right\} .
\end{aligned}
$$

The resultant image of label vectors segments the coregistered image stack where all pixels with the same label vector exhibit the same pattern of change as defined by our representation. It is noted that we are not distinguishing between positive and negative changes (increase or decrease in brightness) at this point in the discussion. In Section IV, we shall see that although this segmentation algorithm can provide insight into the kinds of changes occurring in different parts of the image, its performance depends on the thresholds that, in turn, depend on the value of $p_{0}$.

\section{E. Temporal Filtering}

Temporal filtering is designed to enhance and detect a specific pattern of change. This pattern may have been identified using the temporal segmentation technique described above or have been specified by an image analyst. Temporal filtering combines difference images in ways that emphasize those patterns of change that are expected to be observed when a given activity occurs. In temporal segmentation, the label vector was derived from the detected changes. We now use the label vector as a model to specify the pattern of change of interest and define a measure to determine the degree to which changes in imagery over time match the pattern. One 


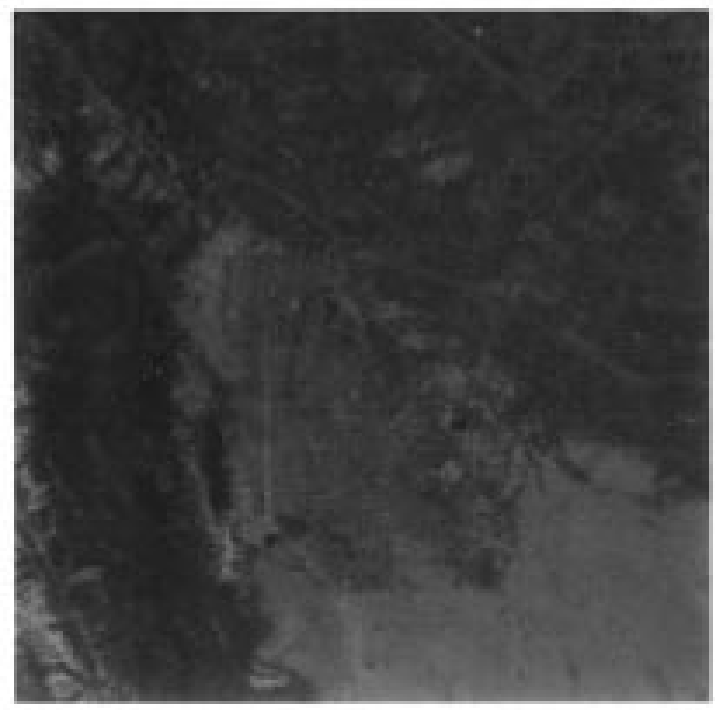

(a)

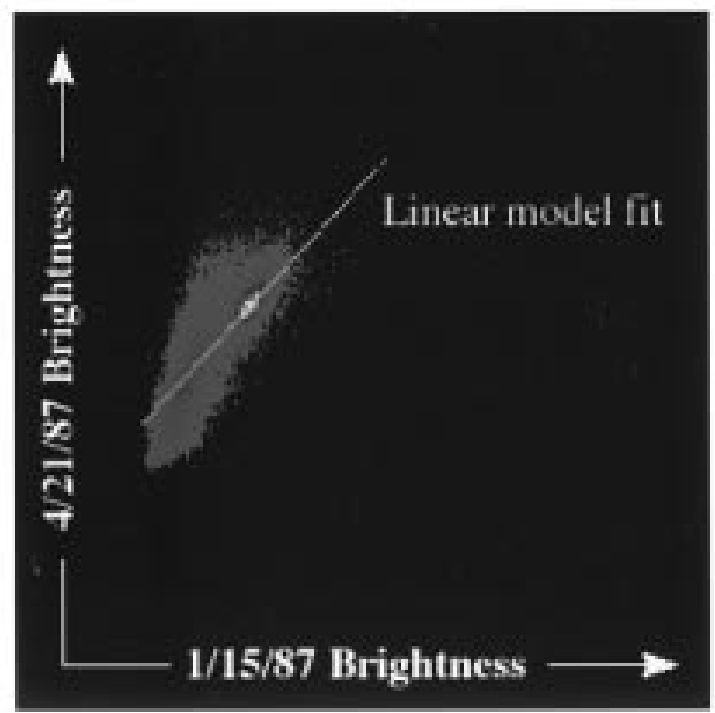

(c)

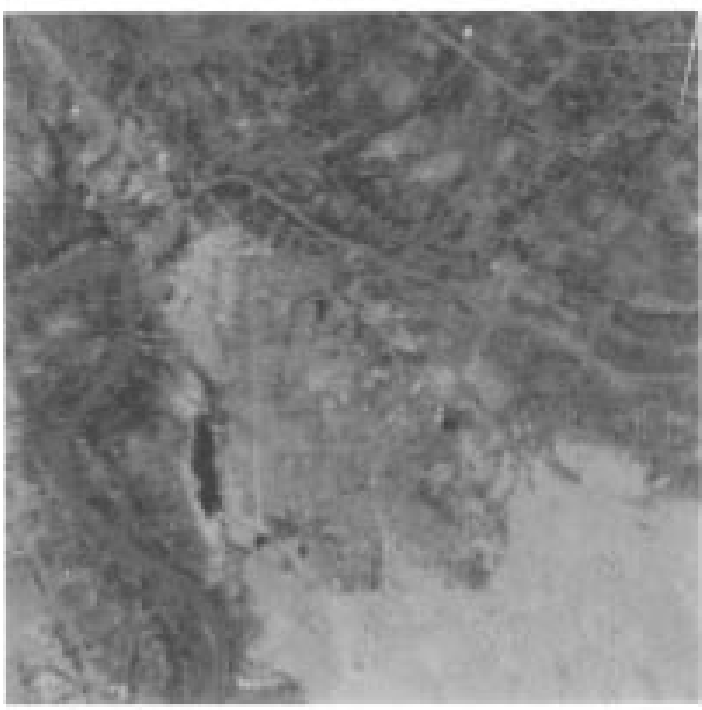

(b)

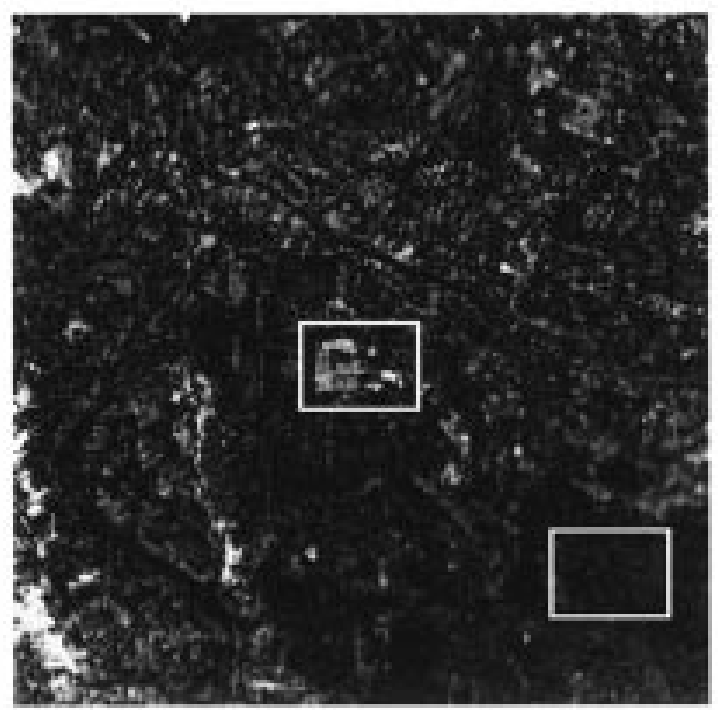

(d)

Fig. 6. Linear prediction algorithm applied to image pair with few changes. (a) 1/15/87 image. (b) 4/21/87 image. (c) Joint distribution. (d) Absolute value of total difference in image.

filter (the absolute difference filter)

$$
a_{\Lambda}(i, j)=\sum_{\lambda_{m} \neq \lambda_{n}}\left|\varepsilon_{m n}(i, j)\right|-\sum_{\lambda_{m}=\lambda_{n}}\left|\varepsilon_{m n}(i, j)\right|
$$

measures the degree to which the absolute value of the differences between images match the pattern of change specified by the label vector $\Lambda$. The filter adds absolute differences that occur when changes are expected and subtracts absolute differences that occur when no changes are expected.

We can do better, however. Up to this point, the values for the different labels in $\Lambda$ were used only to signify that pixels were the same or different; otherwise, the values were arbitrary. We now modify the label vector representation and use the relative values of the labels to represent the direction of change as well. For example, the label vector $\Lambda=[123456]$ describes those changes that are monotonically increasing. The label vector $\Lambda=[345111]$ describes changes that start off at an intermediate value, slowly increase, and then abruptly fall to a low value. The resulting filter (the Delta filter)

$$
\begin{aligned}
d_{\Lambda}(i, j)= & \sum_{\lambda_{m}<\lambda_{n}} \varepsilon_{m n}(i, j)-\sum_{\lambda_{m}>\lambda_{n}} \varepsilon_{m n}(i, j) \\
& -\sum_{\lambda_{m}=\lambda_{n}}\left|\varepsilon_{m n}(i, j)\right|
\end{aligned}
$$

adds differences that occur when positive changes are expected $\left(\lambda_{n}>\lambda_{m}\right)$, subtracts differences that occur when negative changes are expected $\left(\lambda_{n}<\lambda_{m}\right)$, and subtracts the absolute value of the difference when no changes are expected $\left(\lambda_{n}=\right.$ $\left.\lambda_{m}\right)$. Thus, negative differences that occur where positive changes are expected and vice versa, as well as positive or 


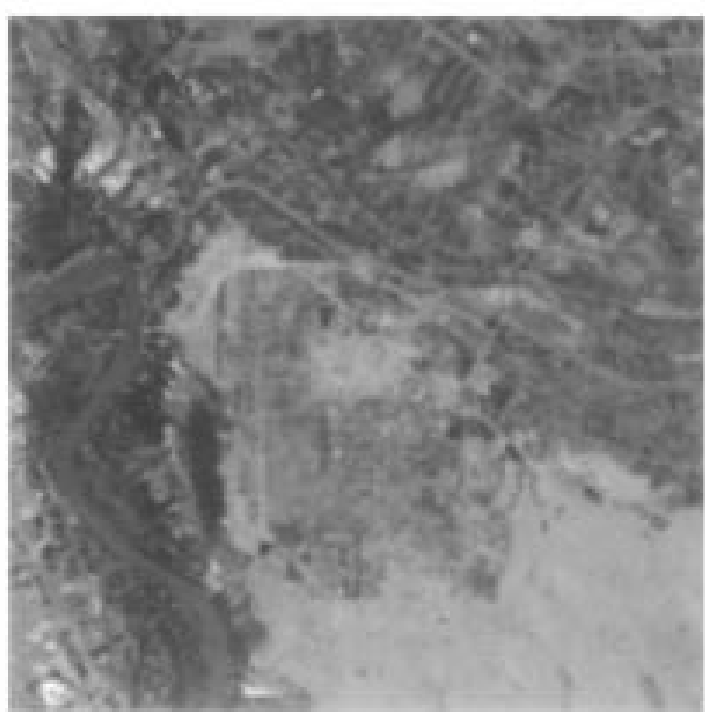

(a)

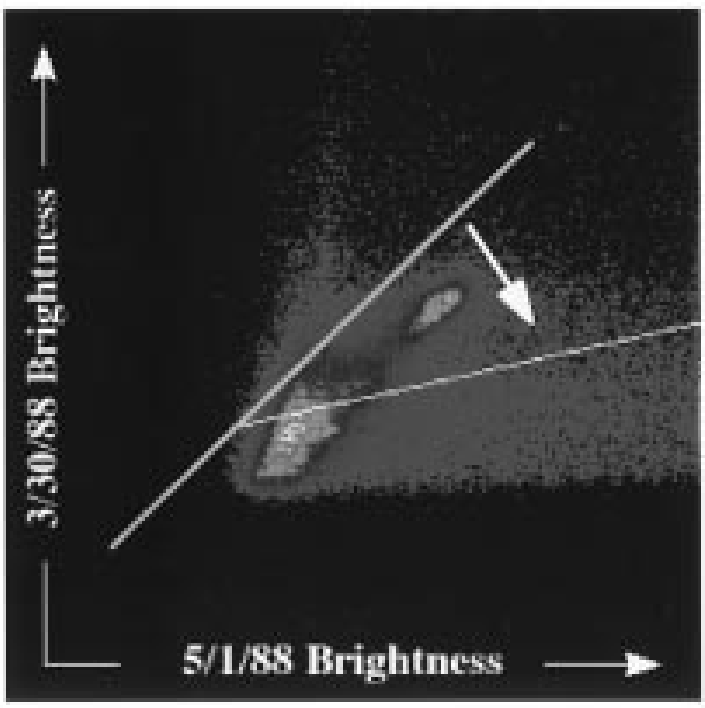

(c)

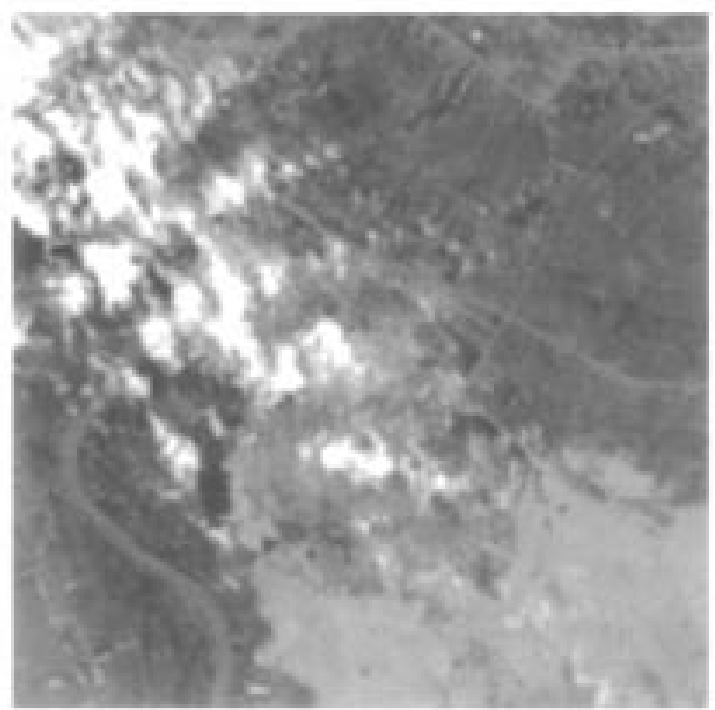

(b)

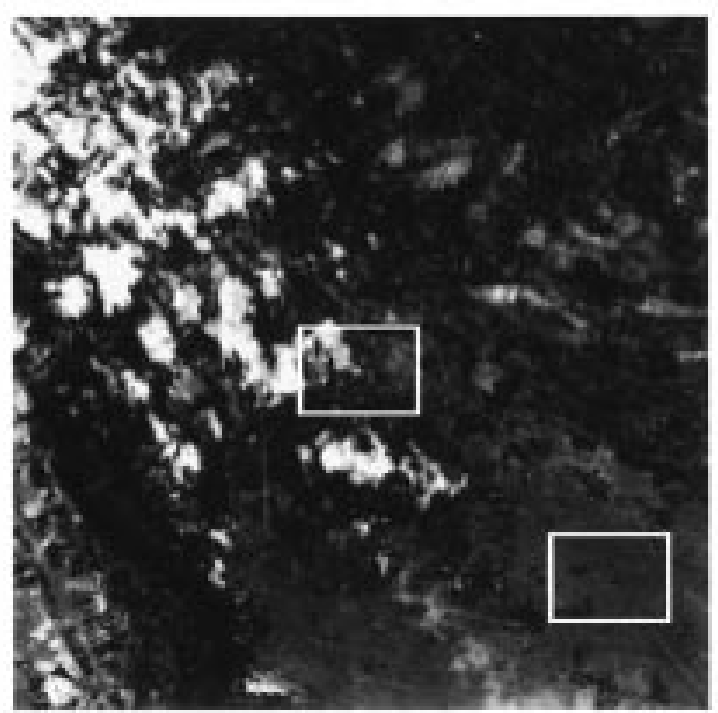

(d)

Fig. 7. Linear prediction algorithm applied to image pair with many changes. (a) 3/30/88 image. (b) 5/1/88 image. (c) Joint distribution. (d) Absolute value of total difference image.

negative differences that occur where no change is expected, reduce the value of the measure.

For a given pattern of change $\Lambda$, the histogram of $d_{\Lambda}(i, j), p(d)=p(d \mid \Lambda) p(\Lambda)+p(d \mid \neg \Lambda) p(\neg \Lambda)$. However, neither the conditionals nor the priors are known in general. Therefore, we threshold the output of the Delta filter

$$
y(i, j)= \begin{cases}1, & d_{\Lambda}(i, j)>\tau \\ 0, & \text { otherwise }\end{cases}
$$

where the threshold $\tau$ satisfies

$$
p_{F A}=\sum_{d>\tau} p(d)
$$

and where $p_{F A}$ is false alarm rate.

\section{E. Postprocessing}

As our method is intended to screen and prioritize areas for rapid review by an image analyst, the binary detection image (13) is postprocessed to produce a list of candidate detections. The binary image is first smoothed by a Gaussian filter to eliminate small isolated detections

$$
z(i, j)=y(i, j) * \frac{1}{2 \pi \sigma^{2}} \exp \left[-\left(i^{2}+j^{2}\right) / 2 \sigma^{2}\right] .
$$

In practice, $2 \sigma$ is chosen to be about the expected size of the activity of interest. Peak locations $\left\{\left(i_{1}, j_{1}\right),\left(i_{2}, j_{2}\right), \cdots\right\}$ in the smoothed detection surface $z(i, j)$ are extracted, rank-ordered in terms of their magnitude $z_{\text {peak }}\left(i_{1}, j_{1}\right)>z_{\text {peak }}\left(i_{2}, j_{2}\right) \cdots$, and used to cue an image analyst to areas in the imagery likely to contain changes of interest. It is noted that because small isolated detections are effectively eliminated by the smoothing 


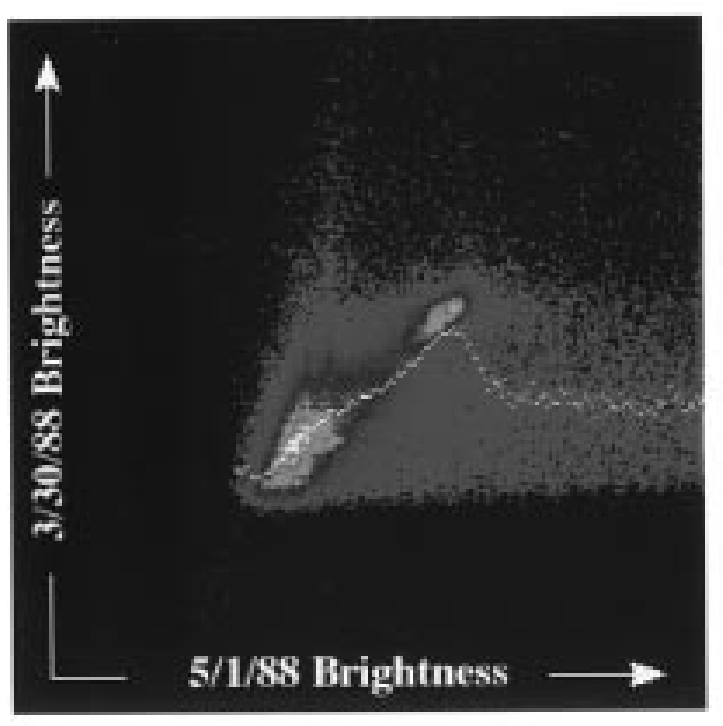

(a)

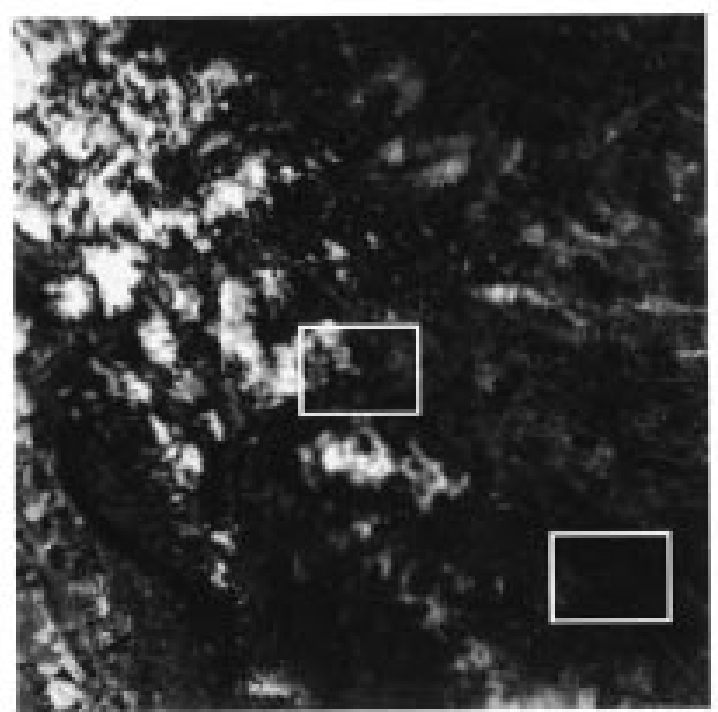

(b)

Fig. 8. Nonlinear prediction algorithm applied to image pair with many changes. (a) Joint distribution. (b) Absolute value of total difference image.

step (15), the actual false alarm rate will be lower than the value specified in (14).

\section{EXPERIMENTAL RESULTS}

\section{A. Study Area}

The study area considered in this paper is a $15000 \mathrm{~km}^{2}$ region in central Iraq (latitude $32^{\circ} 27^{\prime} 47^{\prime \prime}$ to $33^{\circ} 33^{\prime} 16^{\prime \prime}$, longitude $44^{\circ} 16^{\prime} 35^{\prime \prime}$ to $\left.43^{\circ} 19^{\prime} 49^{\prime \prime}\right)$. Ten Landsat thematic mapper (TM) images acquired between June 1986 and July 1990 were coregistered and geocoded. The last image in the series (7/10/90) was used as a reference for registration. The other nine images were registered to this image using a firstorder polynomial warp. Residual registration errors were less than 1 pixel rms. Fig. 3 is a band 7-4-1 false color composite of the 7/10/90 TM image. The coregistered images were $3481 \times 4881$ pixels in size.

Based on open source data, the Iraqi's built a missile-related plant named Taj al-Ma'arik near Latifiyah sometime in the mid to late 1980's [16]-[17]. The approximate location of the plant is indicated in Fig. 3. Fig. 4 shows a series of six Landsat images over the site, which are believed to encompass the missile plant. The first image (Fig. 4(a)) shows the area before construction began in early 1987. The next two images (Fig. 4(b) and (c)) were acquired during the early stages of construction. In this phase of construction, the boundaries of the plant are beginning to take shape, and roads internal to the plant can be seen. The next two images (Fig. 4(d) and (e)) were acquired in what appears to represent the middle phases of construction. Most of the buildings appear to be underway with foundations in, walls up, and in some cases, roofs in place. Light areas surrounding buildings indicate the start of berms. In several of the images, some cloud cover $(<10 \%)$ is present within the scene. A cloud directly over the site is seen in (Fig. 4(e)). The last image (Fig. 4(f)) was acquired after the construction appears to have finished.
TABLE I

Comparison of Linear and Nonlinear Algorithms for Measuring Change

\begin{tabular}{|c|c|c|c|c|c|}
\hline \multicolumn{2}{|c|}{ Thape Dues } & \multicolumn{2}{|c|}{ Lencer Alfonition } & \multicolumn{2}{|c|}{ Nonlace Al aritem } \\
\hline of & $E t_{2}$ & $\overline{\boldsymbol{E}}_{\mathbf{s}}$ & $z_{\text {wats }}$ & 2 & $t_{\text {mens }}$ \\
\hline Th1531 & कास & $\pi$ & & TI & 7 \\
\hline Ywosk & 9008 & 6 & .24 & 7 & $-k$ \\
\hline WSSBS & 7000 & 49 & .10 & 5i & -6 \\
\hline
\end{tabular}

TABLE II

Comparison of Different Band Combinations for Change Detection

\begin{tabular}{|c|c|c|c|c|}
\hline \multicolumn{2}{|c|}{ Bands } & \multicolumn{2}{|c|}{ Average value of total difference } & \multirow{2}{*}{$\begin{array}{l}\text { Figure of } \\
\text { Meril }\end{array}$} \\
\hline Q $t_{1}$ & $\Leftrightarrow t_{1}$ & $\overline{\boldsymbol{E}}_{\operatorname{mat}}$ & $\bar{\varepsilon}_{\text {wo daegs }}$ & \\
\hline T & $T$ & डा & -6 & 85 \\
\hline 4 & 4 & 24 & -7 & 3.4 \\
\hline 7 & 7 & 40 & -3 & 13.3 \\
\hline 6 & 6 & 8 & -2 & 4.0 \\
\hline TCI & $\mathrm{TCl}$ & 81 & -6 & 13.5 \\
\hline $\mathrm{TC} 2$ & $\mathrm{TC}_{2}$ & -12 & -7 & 1.7 \\
\hline 1,7 & 1.7 & 84 & -8 & 10.5 \\
\hline $1,5,7$ & $1,5,7$ & 77 & -5 & 15.4 \\
\hline
\end{tabular}

Fig. 5 plots the average brightness in three of the seven TM bands $(1,4$, and 6$)$ within a region defined by the outer perimeter of the site over time. Band 1 is in the visible portion of the spectrum $(0.45-0.52 \mu \mathrm{m})$, band 4 is in the near infrared $(0.76-0.9 \mu \mathrm{m})$, and band 6 is in thermal infrared (10.4-12.5 $\mu \mathrm{m})$. The brightness in band 1 shows a definite increase over time during the course of the construction. Less change is evident in band 4 . The decrease in brightness in all three bands in the second image is due to the decrease in solar angle in the winter. Overall, the thermal band appears to respond mainly to seasonal variations in temperature.

\section{B. Comparison of Techniques for Measuring Change}

Fig. 6 is an example in which the linear model (3) is used to measure the change between two images using TM band 1 


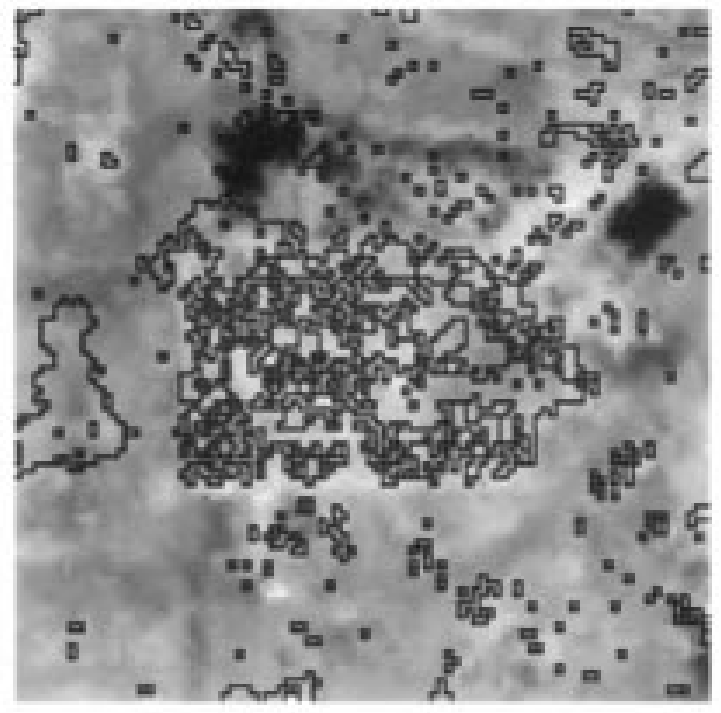

(a)

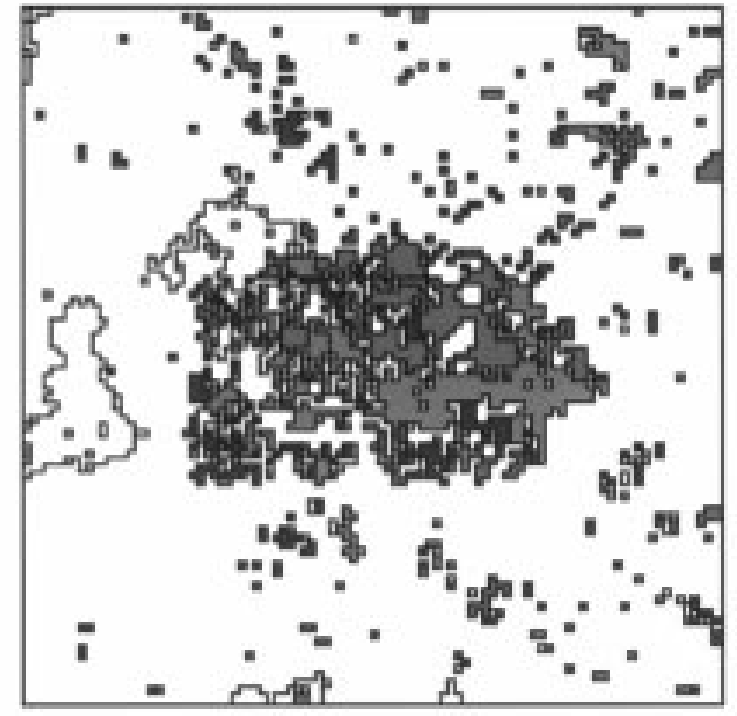

(b)

Fig. 9. Temporal segmentation results for region around Taj al-Ma'arik site. (a) Temporal segmentation. (b) Label vectors associated with construction activity.

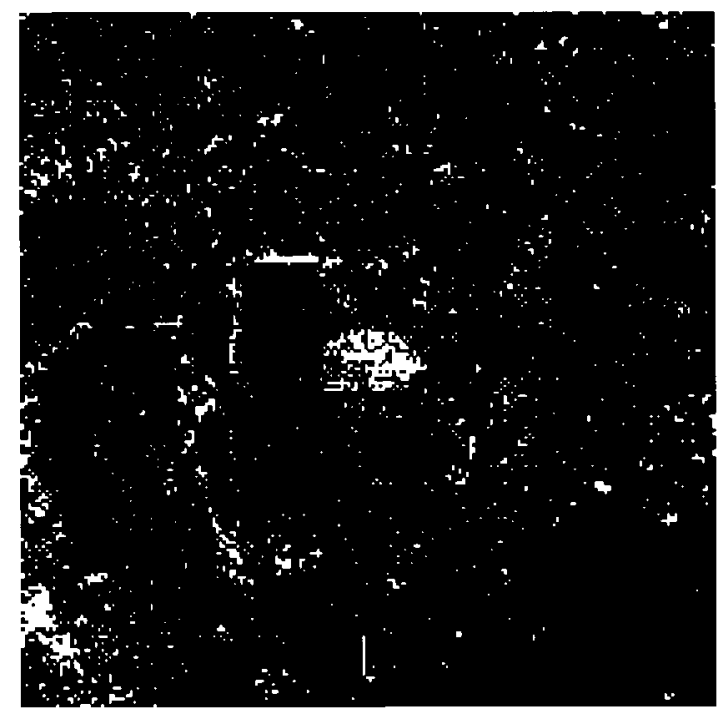

(a)

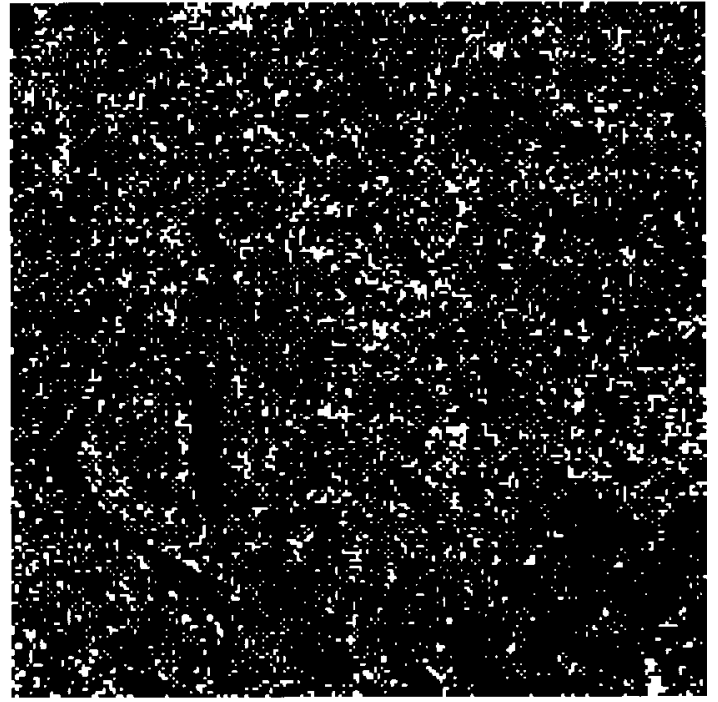

(b)

Fig. 10. Patterns of change related to construction of Taj al-Ma'arik facility derived from two segmentations based on different thresholds. (a) $p_{0}=5 \%$. (b) $p_{0}=25 \%$.

only. The area shown is a $400 \times 400$-pixel region over the Taj al-Ma'arik site. The first image was acquired on 1/15/87, just before the start of construction. The second image was acquired on 4/21/87 during the early stages of construction. The January image (Fig. 6(a)) is darker than the April image (Fig. 6(b)) because of differences in solar angle. The high correlation (low scatter) in the joint histogram (Fig. 6c) implies that there are relatively few changes between the two images. The absolute value of the total difference image (6) is shown in (Fig. 6(d)). Bright areas have either increased or decreased in brightness, and dark areas have remained the same. The linear model adapts easily to the illumination difference between the two images and is able to detect changes within the plant (center box). A region with very little change between June 1986 and July 1990 is identified by the lower right box.

Consider now a second image pair (Fig. 7), where there are significant atmospheric differences between the two images. (Again, we consider only TM band 1.) The two images were taken about a month apart in 3/30/88 and 5/1/88. Except for the clouds, there are relatively few changes on the ground. The clouds in (Fig. 7(b)) significantly increase the scatter in the joint histogram (Fig. 7(c)). They effectively pull the linear model away from the hypothetical regression line, assuming there were no clouds (dotted line) to the actual regression line with clouds (solid line). The absolute value of the total difference image is shown in (Fig. 7(d)) and is dominated 


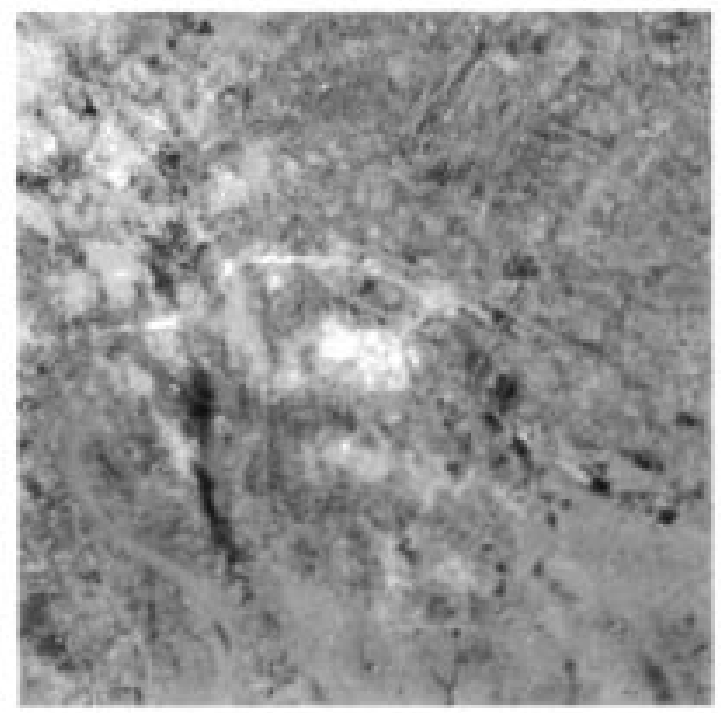

(a)

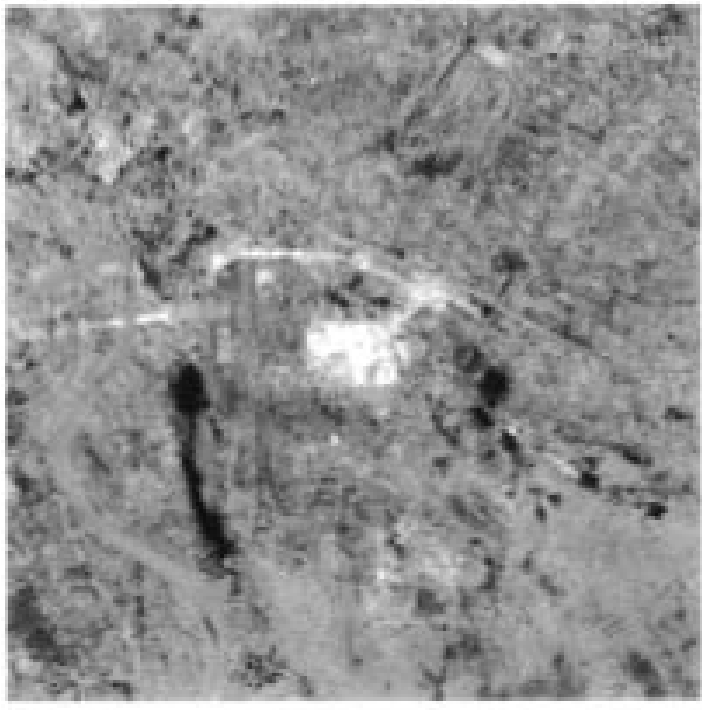

(b)

Fig. 11. Outputs from temporal filters. (a) Absolute different Filter. (b) Delta filter.

TABLE III

DEtection Results versus Number of IMAgES FOR ThreE Model Vectors With $\sigma=20$ AND $p_{F A}=0.25 \%$

\begin{tabular}{|c|c|c|c|c|}
\hline N & Intages Dond & $\Lambda=[11144444]$ & $N=[11344444]$ & $A=[12344444]$ \\
\hline 8 & T22356) & 3 & $T$ & $T$ \\
\hline 7 & 1234567 & 4 & I & i \\
\hline 6 & 123456 & 4 & 3 & 3 \\
\hline 5 & 12345 & 6 & 2 & 1 \\
\hline 4 & 1234 & 6 & 2 & 2 \\
\hline 3 & 23 & 31 & 9 & 9 \\
\hline 2 & 34 & 40 & 49 & 49 \\
\hline
\end{tabular}

TABLE IV

DETECTION RESUltS VERSUS NUMBER OF IMAGES FOR THREE Smoothing Factors with $\Lambda=[11144444]$ AND $p_{F A}=0.25 \%$

\begin{tabular}{|l|l|l|l|l|}
\hline$N$ & Images Used & $\sigma=20$ & $\sigma=10$ & $\sigma=5$ \\
\hline \hline 8 & 12345678 & 3 & 3 & 4 \\
\hline 7 & 1234567 & 4 & 4 & 4 \\
\hline 6 & 123456 & 4 & 4 & 7 \\
\hline 5 & 12345 & 6 & 4 & 9 \\
\hline 4 & 1234 & 6 & 5 & 9 \\
\hline 3 & 234 & 31 & 33 & 49 \\
\hline 2 & 34 & 49 & 79 & 155 \\
\hline
\end{tabular}

by changes dues to the clouds. Although the linear model works well when the amount of change is relatively small (Fig. 6), when there are large changes, in this case due to clouds, the linear model tries to adapt to these changes and shifts the background level. This introduces a residual error that can reduce the detection performance of the algorithm. Such changes are evident in the lower right box, which, as noted above, is over a region that has not changed over this period.

Fig. 8 shows how the nonlinear prediction algorithm can better adapt to large changes. The joint histogram is shown in Fig. 8(a), where the dotted line is a plot of the backward predictor function (5). The function is similar to the hypothetical linear model (Fig. 7(c)) over the part of the joint feature space that does not contain cloud pixel values. A different behavior
TABLE V

Detection Results Versus Number of Images at Two False Alarm Rates with $\Lambda=[1234444]$ AND $\sigma=20$

\begin{tabular}{|l|l|l|l|}
\hline $\mathrm{N}$ & Images Used & $p_{\text {SA }}=0.25 \%$ & $p_{\text {Fa }}=1 \%$ \\
\hline \hline 8 & 12345678 & 1 & 2 \\
\hline 7 & 1234567 & 1 & 3 \\
\hline 6 & 123456 & 3 & 5 \\
\hline 5 & 12345 & 1 & 5 \\
\hline 4 & 1234 & 2 & 6 \\
\hline 3 & 234 & 9 & 9 \\
\hline 2 & 34 & 49 & 54 \\
\hline
\end{tabular}

is seen for the brighter $5 / 1 / 88$ pixel values that are mostly clouds. The spurious change in the lower right introduced by the linear prediction algorithm has been largely eliminated.

Table I gives the average value of the total difference within the site $\bar{\varepsilon}_{\text {site }}$ (center box) and within a region with little or no change $\bar{\varepsilon}_{\text {nochange }}$ (lower right box) for the linear and nonlinear algorithms. Results for three image pairs are shown: 1/15/87-4/21/87 pair in Fig. 6, 3/30/88-5/1/88 pair in Figs. 7 and 8 , and $6 / 5 / 86-7 / 10 / 90$ pair. In all pairs, the differences over the site are comparable. However, over the region with little or no change, the response of the nonlinear technique is lower in magnitude than that of the linear technique overall and is significantly lower in the $3 / 30 / 88-5 / 1 / 88$ pair with cloud cover in the second image.

The above examples used TM band 1. Table II summarizes the performance of the nonlinear change detection algorithm for other spectral bands and features. The table lists $\bar{\varepsilon}_{\text {site }}$ and $\bar{\varepsilon}_{\text {nochange }}$ values computed between the first image (6/5/86) acquired just before the start of construction of Taj al-Ma'arik and the last image (7/10/90) acquired after the facility was completed. As a figure of merit, we computed

$$
\mathrm{FOM}=\bar{\varepsilon}_{\text {site }} / \bar{\varepsilon}_{\text {no change }} \mid
$$

for each spectral band and feature. For this particular facility construction change, certain bands and spectral features are 


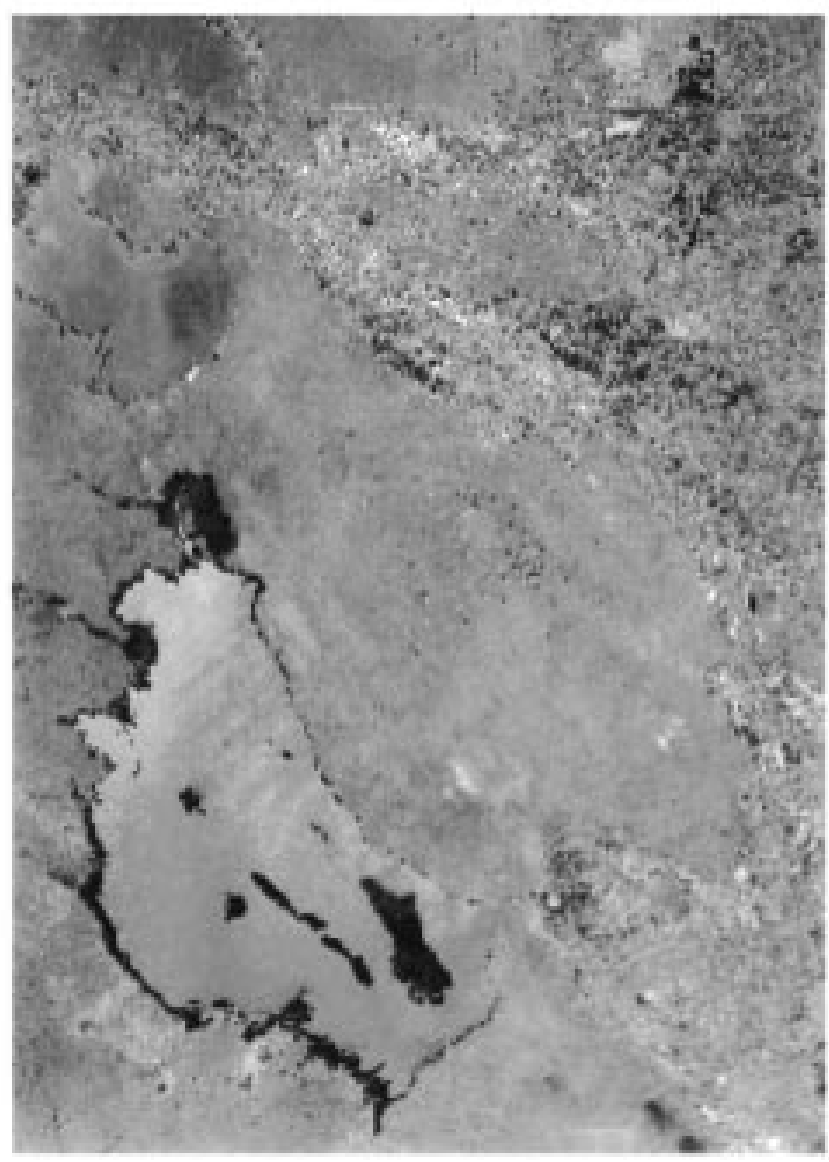

(a)

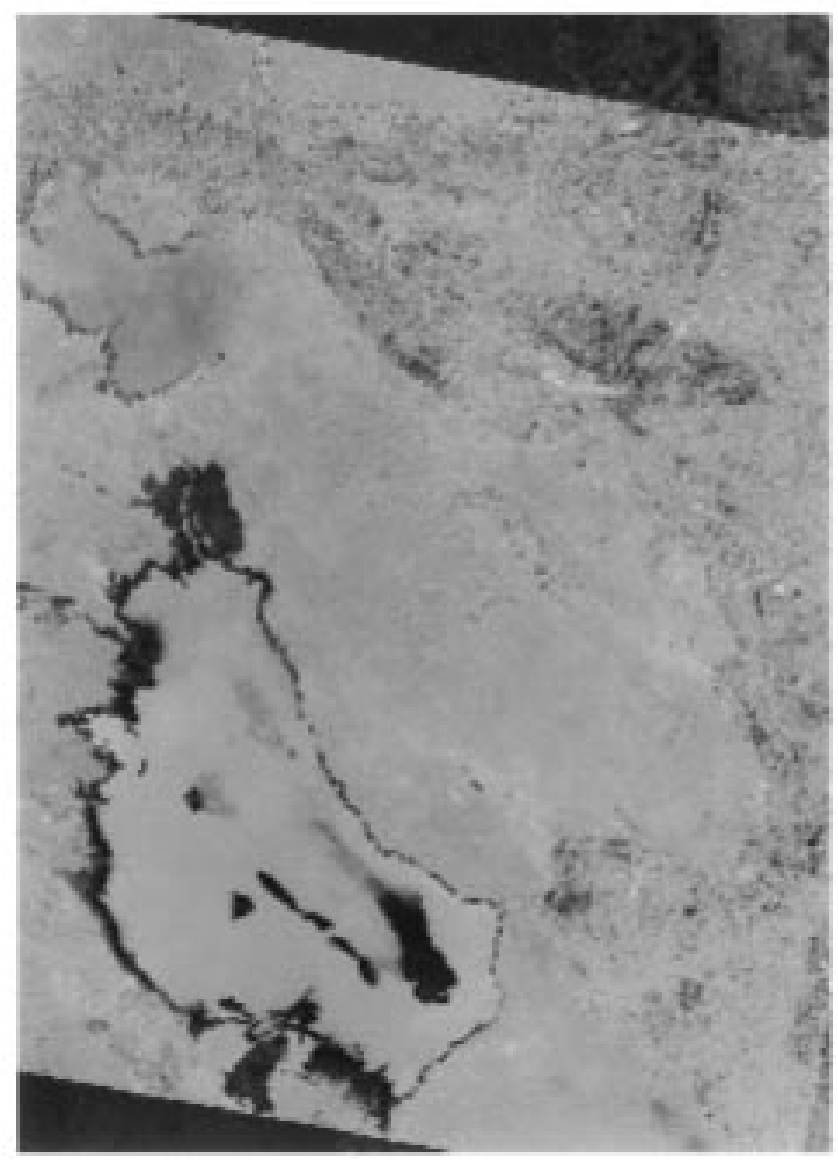

(b)

Fig. 12. Outputs from Delta filter over the full scene. (a) For two images. (b) For eight images.

clearly better than others. TM bands 1 and 7 and the first tasseled cap band (TC1), all of which respond strongly to changes in soil reflectance, give the best results. On the other hand, band 4 and the second tasseled cap band (TC2), both of which respond largely to vegetation, give the worst results. By using more bands (7), the performance improves (provided all the bands respond to the changes of interest).

\section{Temporal Segmentation}

Temporal segmentation was performed on seven of the 10 images (from $1 / 15 / 87$ to $3 / 4 / 90$ ) over the same $400 \times$ 400 pixel region shown in Figs. $6-8$. First, $7 \times 6 / 2=21$ total difference images were computed using the nonlinear prediction algorithm. These images were then thresholded using a value $p_{0}=5 \%$, i.e., $5 \%$ of the pixels in each image were assumed to have changed. The labeling algorithm (10) was then applied to the change images. A total of 138 unique label vectors were identified, with 10 of the 138 label vectors accounting for over $98 \%$ of the pixels. Fig. 9(a) shows the central $100 \times 100$-pixel portion of the segmentation overlaid on the 7/10/90 image as a visual reference. The following three vectors corresponding to visually distinct phases of construction evident in the imagery were extracted interactively: [1 222222$]$, [1 133 333], and [1 114444]. As noted earlier, a cloud passed overhead during the 5/1/88 acqui- sition. Three additional label vectors that include the transient change caused by the cloud are [1 222 522], [1 133 533], and [1 114 544]. These six label vectors are highlighted in Fig. 9(b) with [122 222] and [1 222 522] shown in blue, [113333] and [1 133 533] in green, and [ 1114444$]$ and [ 1114 544] in red.

Fig. 10(a) shows all pixels in the $400 \times 400$ region around the site that match any one of the six label vectors highlighted in Fig. 9(b). The greatest concentration of matches occurs near the site in the center of the image. Fig. 10(b) shows another result computed from the same difference images but using thresholds based on the value $p_{0}=25 \%$ (now, $25 \%$ of the pixels in each image were assumed to have changed). The changes that distinguish the site in Fig. 10(a) are no longer evident in Fig. 10(b). Although the segmentation provides insights into the kinds of changes occurring in different parts of the image, this example suggests a potential weakness in attempting to use the segmentation technique to detect changes directly, namely, that its performance depends critically on the threshold used.

\section{Temporal Filtering}

Fig. 11(a) shows the result obtained by applying the absolute difference filter (11) to the same set of total difference images used above (before thresholding). The filter output depends on the label vector that is used as a model for the 


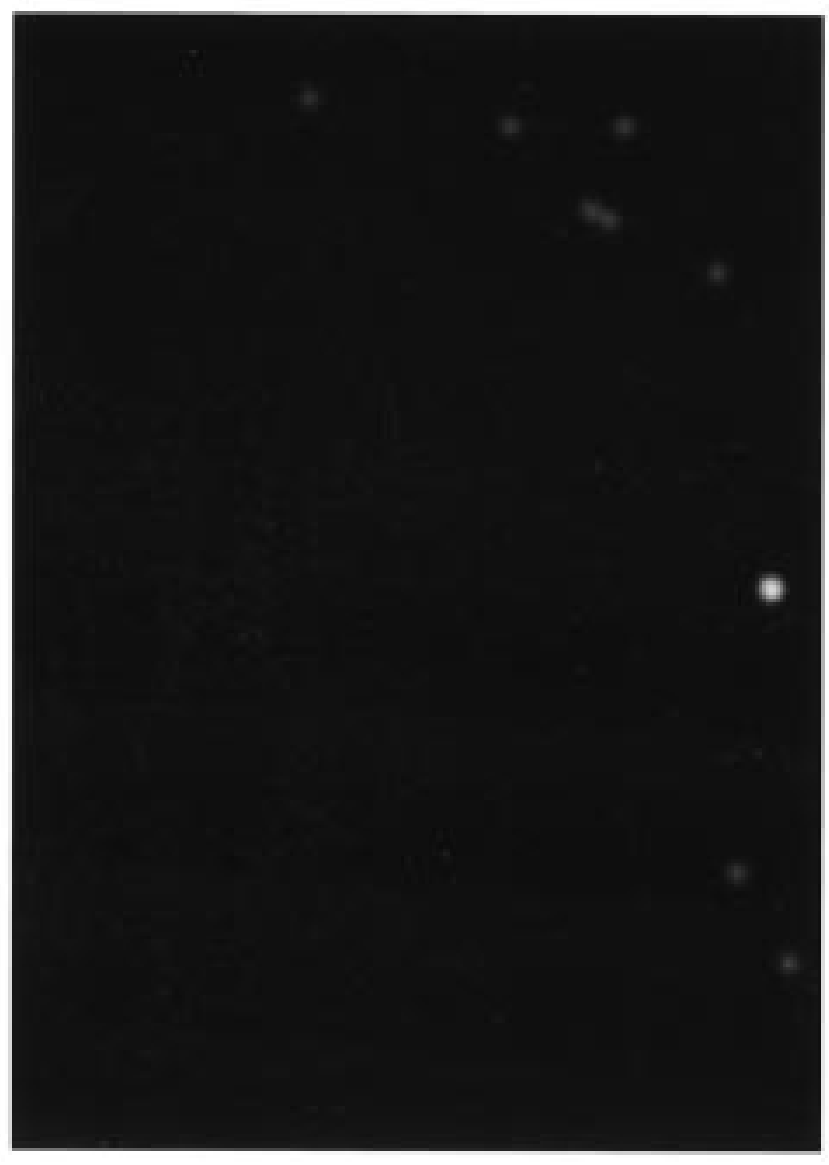

(a)

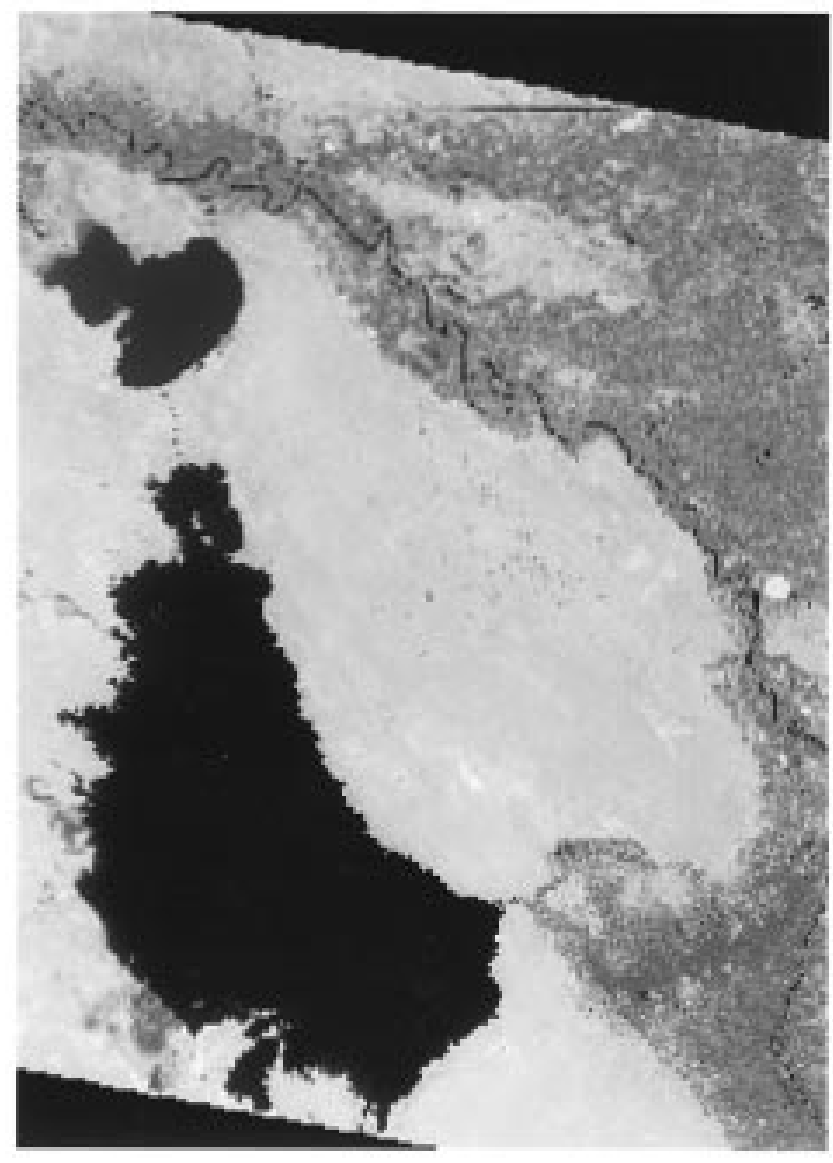

(b)

Fig. 13. Detection result for full scene. (a) Smoothed output from Delta filter. (b) Largest detection highlighted and overlaid.

pattern of change of interest, which in this case was $\Phi=$ [1234444]. This particular filter thus enhances those parts of the image where there are changes in the first, second, and third images and no change in the fourth through seventh image. Fig. 11(b) shows the image generated using the Delta filter (12) using the same model vector. The Delta filter enhances patterns of change that are increasing in brightness in the first through fourth images and then remain relatively constant. It appears to be better able to detect the construction activity with less confusion from the clouds evident in Fig. 11(a).

\section{E. Activity Detection}

A series of activity detection experiments was performed using eight of the $10 \mathrm{TM}$ images from $1 / 15 / 87$ to $3 / 28 / 90$. Based on the experiments in the previous section, we assume that the patterns of change associated with construction activities can be expected to exhibit a monotonic increase in reflectance over the period of construction. We also assume for this experiment that the approximate start date of construction is known but the length of time is not. (In practice, both would be unknown, thus requiring the search to be performed over a sliding window in time.)

Tables III-V summarize detection results versus the number of images used for different model vectors, smoothing factors, and false alarm rates. All results are based on the use of TM band 1 only. The numbers shown are the rank of the detection over the Taj al-Ma'arik facility, i.e., a rank of one means the strongest detection was over the facility (no false alarms). Thus, for a rank of 49 , there were 48 peaks in the smoothed detection surface (15) that were stronger than the one over Taj al-Ma'arik. Given the size are the area processed, the effective false alarm rate would be $48 / 15000 \mathrm{~km}^{2}$ or about one false alarm per $300 \mathrm{~km}^{2}$ for that case. The three model vectors $\Lambda=$ [11 144444], $\Lambda=$ [11344444], and $\Lambda=$ [12344444] correspond to three assumed periods of construction. (Operationally, models corresponding to different assumed lengths of time would have to be applied since the actual period of construction would be unknown). The facility was about $40 \times 60$ pixels in size. The largest smoothing factor is thus intended to enhance either single detections or groupings of detections that are about the size of the facility. Performance for smaller smoothing factors was also measured in order to understand how the performance of the detection algorithm might be different in detecting smaller activities and in cases where the size of the activity is not known in advance. Two false alarm rates were used to determine the sensitivity of the ranking method to thresholds.

Fig. 12 shows the full scene outputs from the Delta filter for two images (Fig. 12(a)) and eight images (Fig. 12(b)) with 
$\Lambda=[1234444], p_{F A}=0.25 \%$ and $\sigma=20$. Fig. 12(a) is the total difference image between the 6/24/87 and 3/30/88 images and represents a two image change detection result. Bright areas indicate an increase in the brightness. In dark areas, the total difference has decreased and includes areas where the water level has risen to cover the land and vegetation has appeared or developed more fully. In Fig. 12(b), there are relatively few bright areas where the Delta filter responds strongly other than over the facility itself. The output from the Delta filter after it has been thresholded and smoothed is shown in Fig. 13(a). The largest detection is highlighted and overlaid on the 7/10/90 image in Fig. 13(b).

Based on open source data, another related facility was constructed about $20 \mathrm{~km}$ south of Taj al-Ma'arik at a slightly later time. As a test of the extendibility of the model for detecting other construction activities occurring at different times, we shifted the model forward in time and applied it to the same stack of eight images. The desired construction activity was detected with 12 false alarms, i.e., it was ranked 13. Seven false alarms were caused by changes in the level of the large lake, three by another construction activity, and two by land clearing for unknown purposes.

\section{CONCLUSION}

A new approach to WAS based on the detection and analysis of changes across two or more images over time was described. Methods for modeling and detecting general patterns of change associated with construction and potentially other kinds of activities that can be observed in remotely sensed imagery were presented. They included a new nonlinear prediction technique for measuring changes between images and temporal segmentation and filtering techniques for analyzing patterns of change over time.

In optical imagery, nonsignificant changes caused by clouds and environmental effects can be expected to occur in an operational environment. It was found that large changes can adversely affect the performance of techniques that use a linear model to measure change. A new nonlinear technique was described and found to be less sensitive to large changes caused by clouds present in one of the images processed. The technique was applied to different spectral bands and features, and its ability to differentiate areas that changed from those that did not was assessed. Bands and features that respond well to changes in soil brightness worked best, whereas those sensitive to vegetation were less effective for the example considered. Methods for selecting the best bands or spectral features to use to detect a particular type of change is an area for future work.

The major contribution of the paper was in the analysis of multiple images over time. Two types of technique were described and compared. The first was based on segmenting the image into distinct patterns of change over time. The second combines difference images to enhance a particular pattern of change. One of the later techniques (Delta filtering) was found to be effective in separating specific patterns of change from general changes. This technique was then used to detect the construction of a known facility over a given period of time. Results derived from a registered set of eight TM images suggest that the method is capable of detecting patterns of change associated with the construction of new facilities with very few false alarms. Under all conditions explored, as the number of images used increased, the number of false alarms was found to decrease dramatically without affecting the detection performance. As a test of the extendibility of the approach, we shifted the model for the pattern of change of interest forward in time and detected another known constructed activity with a relatively small number of false alarms. Additional experiments are currently underway to determine the extent to which other models can be used to detect different kinds of activities and to assess the applicability of our approach to other sensors such synthetic aperture radar.

The promising nature of the results presented in this paper suggest a new approach to WAS that involves ingesting and maintaining registered imagery over regions of interest in large imagery archives. Archives of tens of terabytes or more are currently feasible, given the state of the art in mass storage devices and image database technology. It can be argued that the ability to focus the search on particular patterns of change over time and the resultant processing gain - the reduction in the number of false alarms - which results in using more than two images, justifies the increased computational complexity and storage requirements of our approach.

\section{ACKNOWLEDGMENT}

The author wishes to thank S. McKay for performing all domain research including the identification of the study area, selection of imagery, and detailed analysis of several sites contained in the imagery. He would also like to thank D. Wilson and G. Heberle for registering and geocoding the imagery used in the experiments.

\section{REFERENCES}

[1] S. J. Gee and A. M. Newman, "RADIUS: Automating image analysis through model-supported exploitation," in Proc. DARPA Image Understanding Workshop, Apr. 1993, pp. 185-196.

[2] M. C. Stein, "Fractal image models and object detection," in Proc. Soc Photo-optical Instrum. Eng., vol. 845, 1987, pp. 293-300.

[3] T. Peli, "Multiscale fractal theory and object characterization," J. Opt. Soc. Amer. A, vol. 7, no. 6, June 1990.

[4] A. Rosenfeld, "Automatic detection of changes in reconnaissance data," in Proc. 5th Conv. Mil. Electron., 1961, pp. 492-499.

[5] J. G. Kawamura, "Automatic recognition of changes in urban development from aerial photographs," IEEE Trans. Syst., Man, Cybern., vol. 1, no. 3, July 1971.

[6] K. Price and R. Reddy, "Change detection and analysis in multispectral images," in Proc. 5th Int. J. Conf. Artificial Intell., 1977, pp. 619-625.

[7] C. W. Therrien, T. F. Quatieri, and D. D. Dudgeon, "Statistical modelbased algorithms for image analysis," Proc. IEEE, vol. 74, no. 4, Apr. 1986.

[8] B. G. Lee, V. T. Tom, and M. J. Carlotto, "A signal-symbol approach to change detection," Proc. AAAI, Aug. 1986.

[9] M. J. Carlotto, M. B. Lazaroff, and M. W. Brennan, "Multispectral image processing for environmental monitoring," Proc. SPIE, vol. 1819, pp. 113-124, Nov. 1992

[10] J. L. Engvall, J. D. Tubbs, and Q. A. Holmes, "Pattern recognition of Landsat data based on temporal trend analysis," Remote Sens. Environ. vol. 6, pp. 303-313, 1977. 
[11] F. G. Hall and G. D. Badhwar, "Signature-extendible technology: Global space-based crop recognition," IEEE Trans. Geosci. Remote Sensing, vol. 25, no. 1, Jan. 1987.

[12] G. F. Byrne, P. F. Crapper, and K. K. Mayo, "Monitoring land-cover change by principal component analysis of multitemporal Landsat data," Remote Sens. Environ., vol. 10, pp. 175-184, 1980.

[13] E. P. Crist and R. C. Cicone, "A physically-based transformation of thematic mapper data-The TM tasseled cap," IEEE Trans. Geosci. Remote Sensing, vol. 22, no. 3, May 1984.

[14] A. Papoulis, Probability, Random Variables, and Stochastic Processes. New York: McGraw-Hill, 1965.

[15] M. J. Carlotto, "Non-linear mean-square estimation with applications in remote sensing," Proc. SPIE, vol. 2758, 1996.

[16] K. Timmerman, The Death Lobby. New York: Houghton-Mifflin, 1991.

[17] __, "Iraq rebuilds its military industries," Staff Rep., House Repre sentatives, Committee Foreign Affairs, Subcommittee Int. Security, Int. Organizations, Human Rights, 29 June 1993.

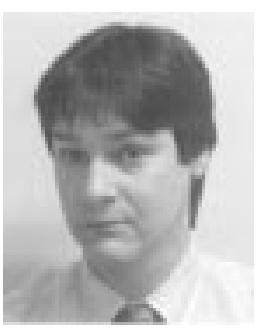

Mark J. Carlotto (SM'90) was born on May 16, 1954 in New Haven, CT. He received the B.S., M.S., and Ph.D. degrees in electrical engineering from Carnegie-Mellon University, Pittsburgh, PA in 1977, 1979, and 1981, respectively.

From 1981 to 1993 , he was with The Analytic Sciences Corp. (TASC), Reading, MA, where he was involved in a variety of projects in the areas of multispectral image processing, object/change detection, image segmentation, scene analysis, geographical information systems, knowledge-based systems, text processing, and data visualization. During this period from 1981 to 1983, he was an Assistant Adjunct Professor in the College of Engineering at Boston University, Boston, MA, where he taught courses in computer architecture and pattern recognition. He is now a senior staff scientist with Pacific-Sierra Research Corporation, Arlington, VA. His current research interests include automated multispectral image classification, terrain feature extraction, change detection and analysis, and image understanding. 